\title{
黄淮麦区小麦粒重基因等位变异的分子鉴定及育种应用
}

\author{
张福彦 ${ }^{1}$ 程仲杰 ${ }^{1} \quad$ 陈晓杰 $^{1}$ 王嘉欢 $^{1} \quad$ 陈 $^{\text {锋 }^{2}}$ 范家霖 $^{1}$ 张建伟 $^{1, *}$
}

杨保安 1 , *

${ }^{1}$ 河南省科学院同位素研究所有限责任公司 / 河南省核农学重点实验室, 河南郑州 $450015 ;^{2}$ 河南农业大学农学院 / 省部共建小麦 玉米作物学国家重点实验室, 河南郑州 450002

摘 要: 采用特异性引物 PCR 扩增方法对 183 份黄淮海麦区的小麦品种(系)的粒重基因 $T a C w i-A 1 、 T a G w 8-B 1$ 和 $T a G S-D 1$ 的等位变异进行分子鉴定, 并结合 2016-2017 和 2017-2018 年度的千粒重表型数据, 分析不同等位变异类 型对小麦粒重的影响, 从而找出优势基因型组合。结果表明, 不同年份间参试品种(系)的千粒重差异达到极显著水平 $(P<0.01) ; T a C w i-A 1$ 位点上发现 $T a C w i-A 1 a$ 和 $T a C w i-A 1 b$ 两种等位变异, 其分布频率分别为 $66.7 \%$ 和 $33.3 \% ; T a G w 8-B 1$ 位点上 $T a G w 8-B 1 a$ 等位变异分布频率较高, 为 $94.5 \%$, 而 $T a G w 8-B 1 b$ 等位变异分布频率极低, 仅为 $5.5 \%$; $T a G S-D 1$ 位点上发现 $T a G S-D 1 a$ 和 $T a G S-D 1 b$ 两种等位变异, 其分布频率分别为 $79.8 \%$ 和 $20.2 \%$ 。不同等位变异组合的品种千 粒重存在显著差异 $(P<0.05)$, 其中具有 3 个高千粒重等位变异组合 $T a C w i-A 1 a / T a G S-D 1 a / T a G w 8-B 1 a$ 品种的平均千粒 重最高, 与具有 $T a C w i-A 1 b / T a G S-D 1 a / T a G w 8-B 1 a$ 品种的平均千粒重差异不显著, 但是显著高于其他组合 $(P<0.05)$ 。 $T a C w i-A 1 a / T a G S-D 1 a / T a G w 8-B 1 b$ 基因型组合小麦品种(系)的平均千粒重最低。TaCwi-A1、TaGw8-B1 和 TaGS-D1 位 点上的不同等位变异均会导致小麦千粒重的显著变化, 其中 $T a G w 8-B 1$ 和 $T a G S-D 1$ 位点上的等位变异对小麦粒重的 影响更为重要。在参试材料中没有发现具有 3 个低千粒重等位变异组合 $T a C w i-A 1 b / T a G S-D 1 b / T a G w 8-B 1 b$ 的品种, 在 7 种不同等位变异组合中, 具有 3 个高千粒重等位变异组合 $T a C w i-A 1 a / T a G S-D 1 a / T a G w 8-B 1 a$ 品种的平均千粒重最高, 是优势基因型组合。

关键词: 小麦; 粒重基因; 功能标记; 等位变异; 千粒重

\section{Molecular identification and breeding application of allelic variation of grain weight gene in wheat from the Yellow-Huai-River Valley}

\author{
ZHANG Fu-Yan ${ }^{1}$, CHENG Zhong-Jie ${ }^{1}$, CHEN Xiao-Jie ${ }^{1}$, WANG Jia-Huan ${ }^{1}$, CHEN Feng ${ }^{2}$, FAN Jia-Lin ${ }^{1}$, \\ ZHANG Jian-Wei, ${ }^{1, *}$, and YANG Bao-An ${ }^{1, *}$ \\ ${ }^{1}$ Isotope Institute Co., Ltd, Henan Academy of Sciences / Henan Key Laboratory of Nuclear Agricultural Sciences, Zhengzhou, 450015, Henan, \\ China; ${ }^{2}$ Agronomy College, Henan Agricultural University / National Key Laboratory of Wheat and Maize Crop Science in Henan Province, Zheng- \\ zhou, 450002, Henan, China
}

\begin{abstract}
Grain weight is one of the most important yield traits in wheat. To accelerate the application process of dominant allelic variation of grain weight gene in wheat breeding, the allelic variation of grain weight gene was identified by using functional markers and the combinations of dominant grain weight genotypes were investigated the allelic variations of grain weight genes TaCwi-A1, TaGw8-B1, and TaGS-D1 in 183 wheat varieties (lines) from the Yellow-Huai-River Valley were identified by PCR amplification with specific primers. To identify the dominant genotype combinations, the effects of different allelic variation
\end{abstract}

\footnotetext{
本研究由河南省小麦产业技术体系建设专项(Z2010-01-04), 河南省重点研发与推广专项(科技攻关)(202102110028), 河南省科学院基本科研 业务专项(190604015)和省部共建小麦玉米作物学国家重点实验室开放课题(30500884)资助。

This study was supported by the Henan Wheat Research System (Z2010-01-04), the Key Research and Development and Promotion Program of Henan Province (202102110028), the Basic Scientific Research Project of Henan Academy of Sciences (190604015), and the National Key Laboratory of Wheat and Maize Crop Science (30500884).

*通信作者(Corresponding authors): 张建伟, E-mail: zjw10308@163.com; 杨保安, E-mail: yangcorn@163.com

第一作者联系方式: E-mail: zhangfuyan704@163.com

Received (收稿日期): 2020-11-03; Accepted (接受日期): 2021-03-19; Published online (网络出版日期): 2021-04-14.

URL: https://kns.cnki.net/kcms/detail/11.1809.S.20210414.1555.006.html
} 
genotypes on wheat grain weight were studied by combining the phenotypic data of the 1000-grain weight (TGW) from 2016-2017 and 2017-2018. The results showed that the difference of TGW between different years was highly significant at $P<$ 0.01. Two alleles, TaCwi-Ala and TaCwi-A1b, were detected at TaCwi-Al locus, with the frequencies of $66.7 \%$ and $33.3 \%$, respectively. The frequency of $T a G w 8-B 1 a$ allele on $T a G w 8-B 1$ locus was up to $94.5 \%$, while the frequency of $T a G w 8-B 1 b$ allele was only 5.5\%. In addition, two alleles, TaGS-DIa and TaGS-DIb, were found in TaGS-D1 locus, and their frequencies were 79.8\% and $20.2 \%$, respectively. Further results indicated that there were significant differences in TGW among different allelic variation combinations at $P<0.05$. Among them, the average TGW of varieties with TaCwi-Ala/TaGS-Dla/TaGw8-Bla genotype was the highest. There was not significant difference in TGW between TaCwi-Ala/TaGS-D1a/TaGw8-B1a and TaCwi-A1b/ TaGS-D1a/TaGw8-B1a genotype, but it was significantly higher than other genotypes at $P<0.05$. The average TGW of TaCwi-Ala/TaGS-Dla/TaGw8-B1b genotype was the lowest. The allelic variations at the loci of TaCwi-A1, TaGw8-B1, and $T a G S-D 1$ all led to significant changes in TGW, and the allelic variations of TaGw8-B1 and TaGS-D1 loci were more important to TGW in wheat. There were no varieties with three low TGW allelic variation combinations $T a C w i-A 1 b / T a G S-D 1 b / T a G w 8-B 1 b$ in the tested materials. Among the seven different allele combinations, the average TGW of three high TGW allelic variation combinations $T a C w i-A 1 a / T a G S-D 1 a / T a G w 8-B 1 a$ was the highest, which was the dominant genotype combination.

Keywords: wheat; grain weight gene; functional marker; allelic variation; TGW

小麦是全球最为重要的粮食作物之一，世界上 约 $40 \%$ 的人口以小麦为主要食粮, 我国小麦的丰歉 直接影响到国家乃至世界粮食安全, 因此高产、稳 产、抗病、优质、广适是现阶段我国小麦育种中最 主要的育种目标之一 ${ }^{[1]}$ 。小麦产量由单位面积穗数、 穗粒数以及粒重三因素构成, 粒重是三要素中遗传 相对稳定的性状, 主要受遗传因子影响且主要受加 性效应的基因控制 ${ }^{[2]}$ 。粒重是影响小麦产量最为重 要因素之一, 粒重在我国不同的小麦生态区均被不 同程度的正向选择和提高, 是现代小麦育种中遗传 改良最为显著的产量性状, 对我国小麦单产水平的 提高做出了较大贡献 ${ }^{[3-4]}$, 但目前关于小麦粒重基因 的遗传基础解析和粒重相关的功能标记在育种中的 应用仍不够深入。

近年来, 随着小麦全基因组序列信息的公布及 现代分子克隆技术的快速发展，关于小麦粒重基因 的克隆和功能标记开发也取得较快发展。Ma 等 ${ }^{[5]}$ 在普通小麦的 $2 \mathrm{AL}$ 染色体上克隆得到细胞壁转化 酶 $T a C w i-A 1$ 基因, 发现该基因的 2 种等位变异, 分 别命名为 $T a C w i-A 1 a$ 和 $T a C w i-A 1 b$, 并根据二者序 列差异开发了一对互补的显性功能标记 CWI21 和 CWI22, 进一步研究发现 $T a C w i-A 1 a$ 变异类型与高 千粒重的品种相关, 而 $T a C w i-A 1 b$ 类型则与低千粒 重的品种相关。之后, Jiang 等 ${ }^{[6]}$ 在普通小麦中克隆 出 $T a C W I$ 基因, 其中 $T a C W I-4 A$ 和 $T a C W I-5 D$ 基因 也都发现了相应的单倍型, 进一步研究发现具有 $H a p-4 A-T$ 和 $H a p-5 D-C$ 单倍型的品种千粒重显著高 于具有 Hap-4A-C 和 Hap- $5 D-G$ 单倍型的品种。 Zhang 等 ${ }^{[7]}$ 利用同源克隆从普通小麦 7DS 染色体上 克隆得到 TaGS-D1 基因, 发现其含有 TaGS-D $1 a$ 和 $T a G S-D 1 b$ 两种等位变异, 根据 $T a G S-D 1$ 基因序列
多态性开发的共显性标记 GS7D, 能够准确检测 $T a G S-D 1 a$ 和 $T a G S-D 1 b$ 类型, 发现拥有不同等位变 异类型品种的千粒重间存在显著性差异。Wang 等 ${ }^{[8]}$ 利用同源克隆技术从普通小麦中克隆了一个控制 籽粒大小的 TaGS5-A1 基因, 该基因被定位在 $3 \mathrm{AS}$ 染色体上, 并发现其至少存在 2 种等位变异, 分别 命名为 TaGS5-A1a 和 TaGS5-A1b。之后, 又在该基 因启动子上游顺式作用元件 $\mathrm{Sp} 1$ 内发现单碱基 $\mathrm{G}$ 的插 入, 这种变化对小麦的粒宽和粒重具有显著影响 ${ }^{[9]}$ 。 Hanif 等 ${ }^{[10]}$ 和 $\mathrm{Hu}$ 等 ${ }^{[11]}$ 利用水稻中的 $T G W 6$ 基因在 普通小麦进行同源克隆, Hanif 等 ${ }^{[10]}$ 克隆得到位于 普通小麦 3AL 染色体上的 TaTGW6-A1 基因, 并发 现该基因在不同品种中存在单核苷酸多态性, 分别 命名为 TaTGW6-Ala 和 TaTGW6-A1b, 而 $\mathrm{Hu}$ 等 ${ }^{[11]}$ 克隆得到 TaTGW6 基因, 并将其定位在普通小麦 $4 \mathrm{AL}$ 染色体上, 且在 $T a T G W 6$ 位点发现 3 种等位变 异类型, 分别命名为: TaTGW6- $a$ 、TaTGW6- $b$ 和 TaTGW6-c, 并开发了共显性标记 TG23, 进一步研 究发现 TaTGW6- $b$ 和 TaTGW6-c 变异类型的品种在 粒径和粒重方面显著高于 TaTGW6- $a$ 类型, 但 TaTGW6- $b$ 和 TaTGW6-c 变异类型在当前品种中分 布概率较低。最近, Yang 等 ${ }^{[12]}$ 同源克隆发现了控制 小麦粒长和粒重的基因 $T a G L 3-5 A$, 被定位在 $5 \mathrm{AL}$ 染色体上, 根据多单核苷酸多态性发现存在 2 种等 位变异, 分别命名为 $T a G L 3-5 A-A$ 和 $T a G L 3-5 A-G$, 后者是小麦粒长和粒重的优势等位变异, 但在我国 小麦中分布频率较低, 认为该类型在我国小麦育种 利用上具有较大潜力。 $\mathrm{Ma}$ 等 ${ }^{[13]}$ 利用同源克隆的方 法获得小麦 TaCYP78A5 基因, 并利用瞬时沉默和 过表达技术揭示 $T a C Y P 78 A 5$ 表达水平与小麦粒重 呈正相关。之后, 司文洁等 ${ }^{[14]}$ 又根据不同小麦品种 
$T a C Y P 78 A 5$ 启动子区序列内 SNP 位点差异开发 TaCYP78A5-2A 启动子区功能标记 CAPS-5Ap, 并 对其进行了验证。Yan 等 ${ }^{[15]}$ 利用同源克隆的方法获 得控制小麦粒重的基因, 命名为 $T a G W 8$, 位于 7BS 上。不同籽粒大小的小麦品种基因多态性分析，发 现 $T a G W 8-B 1$ 基因存在 2 种等位变异, 命名为 $T a G W 8-B 1 a$ 和 $T a G W 8-B 1 b$, 并开发了功能标记 TaGW8-7B, 并发现不同等位变异对小麦粒重有显 著影响。高产和稳产是我国乃至世界小麦育种家永 恒的育种目标，粒重的增加为推动我国乃至世界小 麦单产水平的整体提升做出重要贡献, 而关于小麦 粒重遗传基础解析已成为当前研究热点之一。

截至目前，尽管已有关于小麦粒重基因等位变 异检测的相关报道 ${ }^{[16-17]}$, 但同时对多个粒重基因进 行分子鉴定及粒重功能标记育种应用的研究少之甚 少。本研究以 183 份黄淮海麦区的小麦品种(系)为材 料, 利用功能标记 CWI21、CWI22、TaGW8-7B 以 及 GS7D 分别对 2AL、7BS 和 7DS 染色体上的粒重 基因等位变异进行检测，同时结合 2016-2017 和 2017-2018 年度的千粒重表型数据, 分析不同等位 变异类型对小麦粒重的影响，并找出优势的粒重基 因型组合, 旨在为培育出高产、稳产的小麦新品种 提供一些优异亲本资源, 也为粒重基因功能标记辅 助育种提供参考依据。

\section{1 材料与方法}

\section{1 试验材料}

豫丰11、郑品麦22、百农207、济麦22、石4185、 西农511、西农979、周麦27、周麦32、周麦36、豫 同194、豫农202、鲁原502、良星99、豫同843、丰 德存麦1号、丰德存麦5号等183份小麦品种(系)来自 河南省科学院同位素研究所小麦育种室收集的种质 资源圃, 这些材料主要涵盖了黄淮海麦区近 20 年来
生产上种植的小麦品种(系)。

\section{2 表型测定}

2016-2017和2017-2018年度在河南省科学院 新郑试验基地种植, 每个品种(系)种植 2 行, 行长 2.5 $\mathrm{m}$, 行距 $0.25 \mathrm{~m}$, 每行播 50 粒种子, 待出苗后每行定 苗至30株左右。田间管理同大田生产, 正常成熟后 人工收获, 及时晾晒, 储藏备用。

千粒重测定, 采用德国福弗(pfeuffer)公司生产 的康达得型(contador)全自动数粒仪进行种子计数, 每份材料均匀取 1000 粒, 重复3次, 同时采用常熟市 天量仪器有限责任公司生产的LT1002E型电子天平 进行称重。

\section{3 基因型检测}

每个材料选取 3 粒有代表性的种子, 粉碎后分 别放入 3 个 $2 \mathrm{~mL}$ 离心管中, 根据Chen等 ${ }^{[18]}$ 提取籽粒 DNA的方法, 采用SLS (十二酰肌氨酸钠) 快速提取 小麦籽粒DNA法提取参试材料DNA, 并根据每个品 种(系) 3种功能标记检测判断该品种(系)的基因型。

利用已开发功能标记CWI21、CWI22、TaGW87B和GS7D对所有参试材料中粒重基因 $T a C w i-A 1$ 、 $T a G w 8-B 1$ 和 $T a G S-D 1$ 的等位变异进行分子检测(表 1)。PCR反应体系为 $20 \mu \mathrm{L}$, 主要包括buffer缓冲液 2.5 $\mu \mathrm{L}, \mathrm{Taq}$ DNA 聚合酶(北京天根生化科技有限公司) $\left(2.5 \mathrm{U}_{\mu} \mathrm{L}^{-1}\right) 0.4 \mu \mathrm{L}$, dNTP $\left(200 \mu \mathrm{mol} \mathrm{L}{ }^{-1}\right) 1.0 \mu \mathrm{L}$, 上 下游引物 $\left(10 \mathrm{pmol} \mathrm{L}^{-1}\right)$ 各 $0.5 \mu \mathrm{L}$, 模板 DNA $2.0 \mu \mathrm{L}$, $\mathrm{ddH}_{2} \mathrm{O} 13.1 \mu \mathrm{L}$ 。在Tprofessional standard型PCR仪 (Biometra)中进行扩增, PCR反应程序为 $94^{\circ} \mathrm{C}$ 预变性 $5 \mathrm{~min}, 94^{\circ} \mathrm{C}$ 变性 $30 \mathrm{~s}$, 退火温度具体参照(表1)进行, $72^{\circ} \mathrm{C}$ 延伸 $30 \mathrm{~s}, 30$ 个循环; $72^{\circ} \mathrm{C}$ 延伸 $10 \mathrm{~min}$, 反应程序 结束后取 $5 \mu \mathrm{L}$ PCR扩增产物用 $1.5 \%$ 琼脂糖凝胶电泳 分离、利用GeneGreen Nucleic Acid DyeGeneGreen 核酸染料进行染色, Fire Reader凝胶成像系统下进行 扫描成像, 并保存至计算机以备统计分析。

表 1 检测目的基因等位变异的功能标记及其扩增信息

Table 1 Functional markers and amplification information for detecting alleles of target genes in wheat

\begin{tabular}{|c|c|c|c|c|c|c|}
\hline $\begin{array}{l}\text { 基因 } \\
\text { Genes }\end{array}$ & $\begin{array}{c}\text { 等位变异 } \\
\text { Alleles }\end{array}$ & $\begin{array}{c}\text { 标记引物 } \\
\text { Mark primer }\end{array}$ & $\begin{array}{c}\text { 引物序列 } \\
\text { Primer sequences }\left(5^{\prime}-3^{\prime}\right)\end{array}$ & $\begin{array}{c}\text { 退火温度 } \\
\text { Annealing } \\
\text { temperature }\left({ }^{\circ} \mathrm{C}\right) \\
\end{array}$ & $\begin{array}{c}\text { 扩增片段 } \\
\text { Targeted } \\
\text { fragment (bp) }\end{array}$ & $\begin{array}{l}\text { 参考文献 } \\
\text { Reference }\end{array}$ \\
\hline \multirow[t]{2}{*}{$T a G S-D 1$} & $T a G S-D 1 a$ & GS7D-F & AACTTAGGGAGCGAAAACAA & 58 & 562 & {$[7]$} \\
\hline & $T a G S-D 1 b$ & GS7D-R & CACCAAGACTGGAGATGAAA & & 522 & {$[7]$} \\
\hline \multirow[t]{4}{*}{$T a C w i-A 1$} & TaCwi-Ala & CWI22-F & GGTGATGAGTTCATGGTTAAT & 56 & 402 & {$[5]$} \\
\hline & & CWI22-R & AGAAGCCCAACATTAAATCAAC & & & \\
\hline & $T a C w i-A 1 b$ & CWI21-F & GTGGTGATGAGTTCATGGTTAAG & 56 & 404 & {$[5]$} \\
\hline & & CWI21-R & AGAAGCCCAACATTAAATCAAC & & & \\
\hline \multirow[t]{2}{*}{$T a G w 8-B 1$} & $T a G W 8-B 1 a$ & TaGW8-7B-F & CGCTCATCCATTCCTTCATCG & 60 & 1097 & {$[15]$} \\
\hline & $T a G W 8-B 1 b$ & TaGW8-7B-R & GCTATATGGGTTGGTGTCGC & & 1373 & {$[15]$} \\
\hline
\end{tabular}




\section{4 统计分析}

采用 SPSS 18.0 软件进行数据分析, 对具有不同 粒重基因型的小麦品种(系)间千粒重表型进行差异 显著性分析和方差分析(ANOVA)，并利用 LSD 法进 行多重比较。

\section{2 结果与分析}

\section{1 不同年份间粒重的差异分析}

从表 2 可知，不同年份间供试品种(系)的千粒重 差异达到极显著水平。两个年度间供试品种(系)的千 粒重平均值分别为 $44.11 \mathrm{~g}$ 和 $46.29 \mathrm{~g}$, 变幅分别为 $34.50 \sim 53.35 \mathrm{~g}$ 和 $34.20 \sim 58.45 \mathrm{~g}$, 其变异系数均较高,
分别为 $8.48 \%$ 和 $8.75 \%$ 。可见, 黄淮海地区小麦粒重 的遗传变异非常丰富，具有较大的遗传改良潜力。

\section{2 粒重基因不同等位变异分子检测}

利用显性互补功能标记 CWI21和 CWI22检测 $T a C w i-A 1$ 基因在供试材料中的不同等位变异, 发现 CWI21标记在石4185、郑品麦22、郑育麦16、豫丰 11 、农大 2011 、轮选166、豫农186和淮麦19等61份 材料中可扩增出 $404 \mathrm{bp}$ 的片段, 属于 $T a C w i-A 1 b$ 类型 (图1-B), 而CWI22标记在商麦167、中育1220、涡麦 66、泛育麦17、郑麦 103 、郑麦369和周麦36等 122 个材料中扩增出 $402 \mathrm{bp}$ 的片段, 属于 TaCwi-Ala类型 (图1-A)。

表 2 不同年份中供试材料的千粒重方差分析

Table 2 ANOVA of thousand-grain weight in partial wheat varieties in different year

\begin{tabular}{cccccc}
\hline 年份 & $\begin{array}{c}\text { 千粒重 } \\
\text { Year }\end{array}$ & $\begin{array}{c}\text { 变幅 } \\
\text { Range }\end{array}$ & $\begin{array}{c}\text { 方差 } \\
\text { Variance }\end{array}$ & $\begin{array}{c}\text { 标准差 } \\
\text { Standard deviation }\end{array}$ & $\begin{array}{c}\text { 变异系数 } \\
\text { Coefficient of variation (\%) }\end{array}$ \\
\hline $2016-2017$ & 44.11 & $34.50-53.35$ & $14.00^{* *}$ & 3.74 & 8.48 \\
$2017-2018$ & 46.29 & $34.20-58.45$ & $16.42^{* *}$ & 4.05 & 8.75 \\
\hline
\end{tabular}

"*表示差异达到 $P<0.01$ 显著水平。 ${ }^{* *}$ Significant at $P<0.01$.

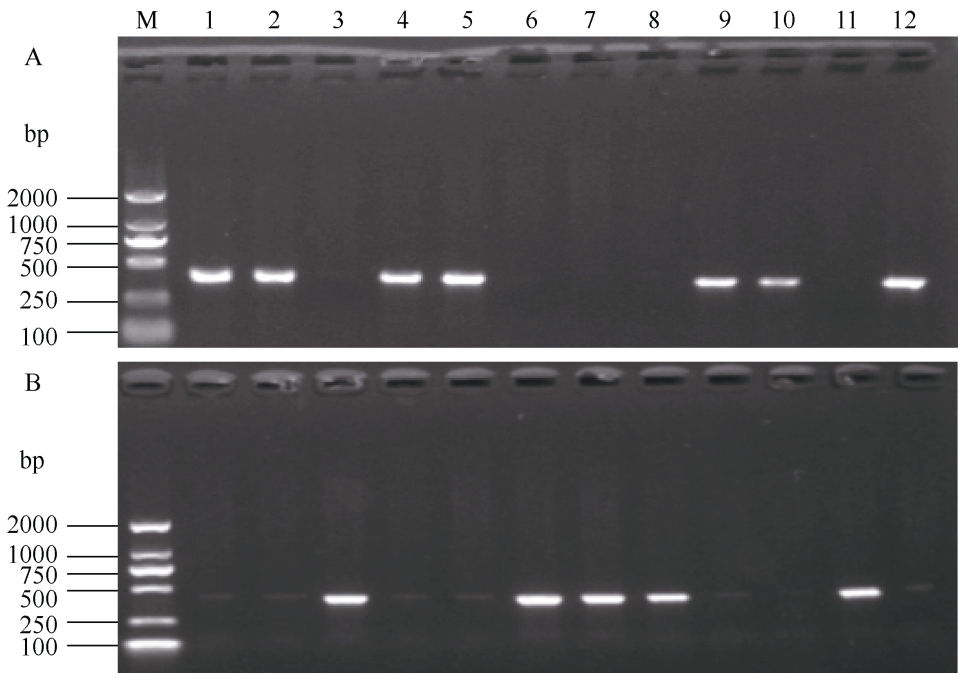

图 1 标记 CWI21 (A)和 CWI22 (B)在部分小麦品种中扩增结果

Fig. 1 Amplification of $\mathrm{TaCwi}$-A1 alleles by functional markers CWI21 (A) and CWI22 (B) in partial wheat varieties M: DL2000; 1：石 $4185 ; 2$ : 郑品麦 22; 3：商麦 $167 ; 4$ ：郑育麦 $16 ; 5$ ：豫丰 $11 ; 6$ ：中育 $1220 ; 7$ : 涡麦 66; 8：泛育麦 17; 9：豫农 186; 10: 赛德麦 1 号; 11: 郑麦 369; 12: 淮麦 19 。

M: DL2000; 1: Shi 4185; 2: Zhengpinmai 22: 3: Shangmai 167; 4: Zhengyumai 16; 5: Yufeng 11; 6: Zhongyu 1220; 7: Guomai 66; 8: Fanyumai 17; 9: Yunong 186; 10: Saidemai 1; 11: Zhengmai 369; 12: Huaimai 19.

利用共显性功能标记TaGW8-7B对所有参试材 料进行分子检测(图2), 发现在商麦167、金农 518 、 中新16、豫丰11、皖科06725、轮选166、泉麦29、 农大 2011 、郑麦1860等173份材料中扩增出 $1097 \mathrm{bp}$ 的片段，属于 $T a G W 8-B 1 a$ 类型，而在温麦6号、郑麦 518、豫农202、石84-7111、淮麦19等10份材料中扩 增出 $1373 \mathrm{bp}$ 的片段, 属于 $T a G W 8-B 1 b$ 类型。
利用共显性标记GS7D对 183 份小麦品种(系) 进行检测(图3), 发现在鲁原502、誉兴0369、轮选 987、周8425B、兰考906、周麦27、西农511、中 育1220、豫丰11等146份材料中扩增出 $562 \mathrm{bp}$ 的片 段，属于 $T a G S-D 1 a$ 类型，而在豫农 186 、赛德麦 1 号、龙科 1221 、中育 9302 等 37 份材料中可扩增出 $522 \mathrm{bp}$ 的片段，属于 $T a G S-D 1 b$ 类型。 


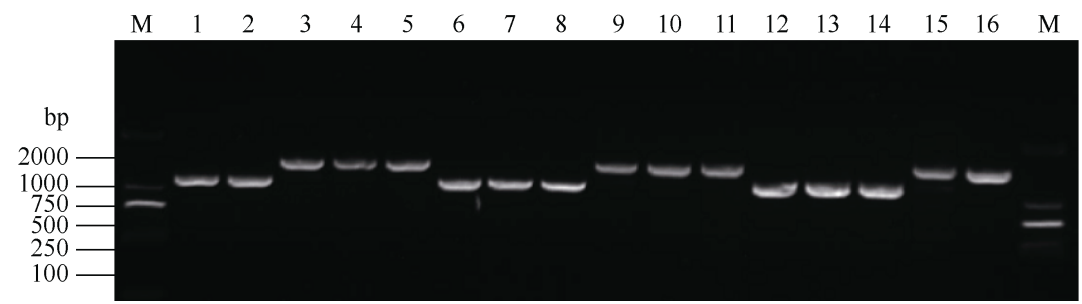

图 2 TaGW8-7B 标记在部分小麦品种中扩增结果

Fig. 2 Amplification of TaGW8 genes by functional marker TaGW8-7B in partial wheat varieties

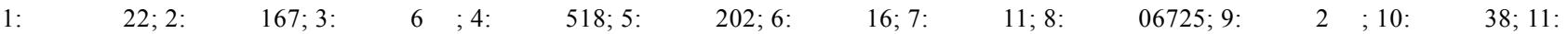
石 84-7111; 12: 丰德存麦 5 号; 13: 良星 99; 14: 洛麦 28; 15: 淮麦 19; 16: 百农 3217; M: DL2000。

1: Zhengpinmai 22; 2: Shangmai 167; 3: Wenmai 6; 4: Zhengmai 518; 5: Yunong 202; 6: Zhongxin 16; 7: Yufeng 11; 8: Wanke 06725; 9: Yumai 2; 10: Jimai 38; 11: Shi 84-7111; 12: Fengdecunmai 5; 13: Liangxing 99; 14: Luomai 28; 15: Huaimai 19; 16: Bainong 3217; M: DL2000.

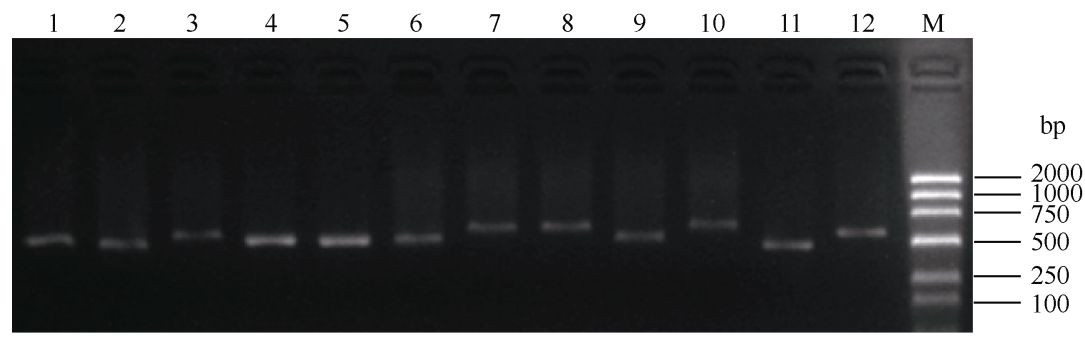

图 3 GS7D 标记检测部分小麦品种

Fig. 3 Molecular detection for partial wheat varieties by GS7D

1: 豫农 186; 2: 赛德麦 1 号; 3: 鲁原 502; 4: 郑麦 366; 5: 中育 9302; 6: 龙科 1221； 7：㵙兴 0369; 8：豫丰 11；9：农大 2011；10：郑品 麦 8 号; 11: 高麦 6 号; 12: 丰德存麦 5 号; M: DL2000。

1: Yunong 186; 2: Saidemai 1; 3: Luyuan 502; 4: Zhengmai 366; 5: Zhongyu 9302; 6: Longke 1221; 7: Puxing 0369; 8: Yufeng 11; 9: Nongda 2011; 10: Zhengpinmai 8; 11: Gaomai 6; 12: Fengdecunmai 5; M: DL2000.

\section{3 粒重基因不同等位变异的分布及其对小麦} 粒重的影响

在所有参试材料中, $T a C w i-A 1 、 T a G w 8-B 1$ 和 $T a G S-D 1$ 位点具上均发现 2 种等位变异。从表 3 可知, $T a C w i-A 1$ 位点上鉴定发现 183 份参试材料中 $T a C w i-$ $A 1 a$ 等位变异的分布频率为 $66.67 \%, T a C w i-A 1 b$ 为 $33.33 \%$, 同时利用SPSS 18.0 软件对不同等位变异 对应的千粒重进行方差分析, 发现具有 $T a C w i-A 1 a$ 等位变异类型品种的千粒重显著高于具有 $T a C w i$ $A 1 b$ 等位变异类型品种的千粒重 $(P<0.05), T a C w i-$
$A 1 a$ 为优异等位基因。 $T a G w 8-B 1$ 位点上鉴定所有参 试材料中 $T a G w 8-B 1 a$ 等位变异分布频率较高, 为 $94.54 \%$, 而 $T a G w 8-B 1 b$ 等位变异分布频率极低，仅 为 $5.46 \%$, 方差分析发现具有 $T a G w 8-B 1 a$ 等位变异 类型品种的千粒重与具有 $T a G w 8-B 1 b$ 等位变异类型 品种的千粒重, 二者差异达到极显著水平 $(P<0.01)$, $T a G w 8-B 1 a$ 为优异等位基因。 $T a G S-D 1$ 位点上鉴定 所有参试材料中 $T a G S-D 1 a$ 等位变异分布频率为 $79.78 \%, T a G S-D 1 b$ 为 $20.22 \%$ ，方差分析发现具有 $T a G S-D 1 a$ 等位变异类型品种的千粒重与具有

表 3 粒重基因的不同等位变异对小麦千粒重影响

Table 3 Effects of alleles of different grain weight genes on the thousand-grain weight (TGW) of wheat varieties

\begin{tabular}{|c|c|c|c|c|c|c|}
\hline $\begin{array}{l}\text { 基因位点 } \\
\text { Gene locus }\end{array}$ & $\begin{array}{c}\text { 等位变异 } \\
\text { Allele }\end{array}$ & $\begin{array}{c}\text { 品种数量 } \\
\text { Number of varieties }\end{array}$ & $\begin{array}{c}\text { 频率 } \\
\text { Frequency (\%) }\end{array}$ & $\begin{array}{c}2016 \text { 年千粒重 } \\
\text { TGW in } 2016(\mathrm{~g})\end{array}$ & $\begin{array}{c}2017 \text { 年千粒重 } \\
\mathrm{TGW} \text { in } 2017(\mathrm{~g})\end{array}$ & $\begin{array}{c}\text { 均值 } \\
\text { Mean }(\mathrm{g})\end{array}$ \\
\hline \multirow[t]{2}{*}{ TaCwi-Al } & TaCwi-Ala & 122 & 66.7 & $44.46^{*}$ & $46.61^{*}$ & $45.54^{*}$ \\
\hline & $T a C w i-A 1 b$ & 61 & 33.3 & 43.40 & 45.65 & 44.52 \\
\hline \multirow[t]{2}{*}{$T a G w 8-B 1$} & $T a G w 8-B 1 a$ & 173 & 94.5 & $44.27^{* *}$ & $46.51^{* *}$ & $45.39^{* *}$ \\
\hline & $T a G w 8-B 1 b$ & 10 & 5.5 & 41.34 & 42.46 & 41.90 \\
\hline \multirow[t]{2}{*}{$T a G S-D 1$} & $T a G S-D l a$ & 146 & 79.8 & $44.44^{* *}$ & $46.71^{* *}$ & $45.58^{* *}$ \\
\hline & $T a G S-D 1 b$ & 37 & 20.2 & 42.79 & 44.63 & 43.71 \\
\hline
\end{tabular}

"和 ${ }^{* *}$ 分别表示差异达到 $P<0.05$ 和 $P<0.01$ 显著水平。

* and ${ }^{* *}$ indicate significant difference at $P<0.05$ and $P<0.01$, respectively. 
$T a G S-D 1 b$ 等位变异类型品种的千粒重, 二者差异 也达到极显著水平 $(P<0.01), T a G S-D 1 a$ 为优异等位 基因。说明 $T a C w i-A 1 、 T a G w 8-B 1$ 和 $T a G S-D 1$ 位点 上不同等位变异均会导致小麦千粒重的显著变化, 而 $T a G w 8-B 1$ 和 $T a G S-D 1$ 位点上的等位变异对粒重 具有更为重要的影响。

\section{4 不同基因型组合与小麦粒重的关系}

利用功能标记 CWI21、CWI22、TaGW8-7B 和 GS7D 对所有参试材料进行检测。在 $T a C w i-A 1$ 、 $T a G w 8-B 1$ 和 TaGS-D1 位点上共发现 TaCwi-Ala/ TaGS-D1a/TaGw8-B1a、TaCwi-A1b/TaGS-D1a/ TaGw8-B1a、TaCwi-A1b/TaGS-D1b/TaGw8-B1a、 TaCwi-Ala/TaGS-D1b/TaGw8-B1a、TaCwi-Alb/TaGSD1a/TaGw8-B1b、TaCwi-Ala/TaGS-D1b/TaGw8-B1b 和 TaCwi-A1 a/TaGS-Dla/TaGw8-B1b 共 7 种不同的等 位变异组合，分别占参试材料的 $52.5 \% 、 23.5 \%$ 、
$7.1 \% 、 11.5 \% 、 2.7 \% 、 1.6 \%$ 和 $1.1 \%$ 。从表 4 可知，具 有不同等位变异组合品种的千粒重间存在显著差 异。具有 3 个高千粒重等位变异组合 $T a C w i-A l a /$ $T a G S-D 1 a / T a G w 8-B 1 a$ 品种的平均千粒重最高, 为 $44.69 \mathrm{~g}$, 与具有 $T a C w i-A 1 b / T a G S-D 1 a / T a G w 8-B 1 a$ 品 种的平均千粒重差异不显著, 但是显著高于其他组 合; 在参试材料中没有发现具有 3 个低千粒重等位 变异组合 $T a C w i-A 1 b / T a G S-D 1 b / T a G w 8-B 1 b$ 的品种; 具有 TaCwi-Alb/TaGS-D1b/TaGw8-Bla、TaCwi-Alal TaGS-D1b/TaGw8-B1a、TaCwi-Alb/TaGS-D1a/TaGw8$B 1 b$ 和 $T a C w i-A 1 a / T a G S-D 1 b / T a G w 8-B 1 b$ 等位变异组 合品种 (系)间的平均千粒重差异也不显著; 而 $T a C w i-A 1 a / T a G S-D 1 a / T a G w 8-B 1 b$ 基因型组合小麦品 种(系)的平均千粒重最低, 仅为 $41.39 \mathrm{~g}$ 。可见, $T a C w i-A 1 、 T a G w 8-B 1$ 和 $T a G S-D 1$ 位点上不同等位 变异组合会引起小麦粒重的显著变化。

表 $4 T a C w i-A 1 、 T a G S-D 1$ 和 $T a G w 8-B 1$ 位点上不同等位变异组合对小麦千粒重的影响

Table 4 Effects of alleles of allelic variation combination of $T a C w i-A 1, T a G S-D 1$, and $T a G w 8-B 1$ on the thousand-grain weight (TGW) in wheat varieties

\begin{tabular}{|c|c|c|c|c|c|c|}
\hline $\begin{array}{c}\text { 等位变异组合 } \\
\text { Allelic variation combination }\end{array}$ & $\begin{array}{c}\text { 品种数量 } \\
\text { Number of varieties }\end{array}$ & $\begin{array}{c}\text { 频率 } \\
\text { Frequency }(\%)\end{array}$ & $\begin{array}{c}\text { 表型 }^{\dagger} \\
\text { Phenotype }^{\dagger}\end{array}$ & $\begin{array}{l}2016 \text { 年千粒重 } \\
2016 \mathrm{TGW}(\mathrm{g})\end{array}$ & $\begin{array}{l}2017 \text { 年千粒重 } \\
2017 \mathrm{TGW}(\mathrm{g})\end{array}$ & $\begin{array}{c}\text { 均值 }^{\star} \\
\text { Mean }(\mathrm{g})^{\ddagger}\end{array}$ \\
\hline TaCwi-Ala/TaGS-D1a/TaGw8-B1a & 96 & 52.5 & $\mathrm{H}$ & 43.67 & 45.71 & $44.69 \mathrm{a}$ \\
\hline TaCwi-A1b/TaGS-D1a/TaGw8-B1a & 43 & 23.5 & $\mathrm{H}$ & 43.28 & 45.73 & $44.50 \mathrm{a}$ \\
\hline TaCwi-A1b/TaGS-D1b/TaGw8-B1a & 13 & 7.1 & M & 41.56 & 43.53 & $42.54 \mathrm{~b}$ \\
\hline TaCwi-Ala/TaGS-D1b/TaGw8-B1a & 21 & 11.5 & M & 41.61 & 43.39 & $42.50 \mathrm{~b}$ \\
\hline TaCwi-A1b/TaGS-D1a/TaGw8-B1b & 5 & 2.7 & ML & 41.69 & 42.90 & $42.30 \mathrm{~b}$ \\
\hline TaCwi-A1a/TaGS-D1b/TaGw8-B1b & 3 & 1.6 & ML & 41.13 & 42.92 & $42.03 \mathrm{bc}$ \\
\hline TaCwi-Ala/TaGS-D1a/TaGw8-B1b & 2 & 1.1 & $\mathrm{~L}$ & 41.18 & 41.60 & $41.39 \mathrm{c}$ \\
\hline
\end{tabular}

${ }^{\dagger} \mathrm{H}$ : 高千粒重; M: 中等千粒重; ML: 中低千粒重; L: 低千粒重。 ${ }^{\ddagger}$ 不同小写字母表示在 $P<0.05$ 水平显著差异。

${ }^{\dagger} \mathrm{H}$ : high thousand-grain weight; M: medium thousand-grain weight; ML: medium low thousand-grain weight; L: low thousand-grain weight. ${ }^{\ddagger}$ Different lowercase letters indicate significant difference at $P<0.05$.

\section{3 讨论}

小麦产量由单位面积穗数、穗粒数以及千粒重 三因素构成，其中穗粒数的增加是建立在单位面积 穗数减少的基础上，而千粒重的增加则是相对独立 的, 且受遗传特性影响最大, 其遗传力高达 $89 \%{ }^{[19]}$ 。 $\mathrm{Wu}$ 等 ${ }^{[20]}$ 研究发现 1945 年至 2010 年以来我国不同 的小麦主产区小麦产量平均每年增产幅度都在 $0.33 \% \sim 1.42 \%$ 之间，而粒重和单穗重的显著增加对 小麦产量的持续增长起到了举足轻重的作用。 Rasheed 等 ${ }^{[21]}$ 研究表明, 在普通小麦中目前已知的 有 TaCwi-2A、TaSus-2B、TaCKX6-3D、TaGS-D1、 $T a C K X 6 a 02$ 和 $T a G w 2-6 A$ 等控制粒长、粒宽、粒重 的基因均具有正向遗传效应，且对小麦粒重高低具
有极其重要的影响。同时, 除了基因型影响外，脱落 酸、赤霉素等内源激素以及光照、温度、水分、养 分等外界环境等多种因素对小麦粒重也产生重要影 响。本文仅对粒重控制基因对小麦粒重的影响以及利 用粒重功能标记进行育种应用进行了研究和讨论。

小麦粒重是一个受多基因控制的复杂数量性状, 与其相关的大多数 QTL 位点的表型贡献率较小, 重 复性较差, 且较为分散。Gupta 等 ${ }^{[22]}$ 对小麦粒长、粒 宽和千粒重的 QTL 遗传定位的研究结果进行了系统 总结, 发现受亲本遗传背景差异、QTL 作图方法、 标记类型等因素的影响, 在不同的作图群体中小麦 千粒重的 QTL 定位的结果存在较大的差异, 且远远 不能满足分子标记辅助选择育种和粒重相关基因克 隆的需要。随着图位克隆、同源克隆等现代生物学 
技术和标记开发技术的快速发展, 目前已先后克隆 出 TaGS-D1、TaTGW6、TaGW8-B1、TaGL3-5A、 TaSus2-B1、TaCwi-A1、TaCWI-D1、TaCKX6-D1 和 $T a C Y P 78 A 5$ 等小麦粒重形成的相关基因, 并开发出 GS7D、TG23、TaGW8-7B、TaSus2-2B tgw $_{\text {、 CWI22、 }}$ CWI21 和 TKX3D 等相应的功能标记, 可快速准确 鉴定不同小麦品种中控制粒重的相关基因及其变异 类型的分布情况。相吉山等 ${ }^{[23]}$ 和刘永伟等 ${ }^{[24]}$ 利用 CWI22、CWI21 标记分别对新疆小麦品种资源和黄 淮麦区的小麦品种资源的 $T a C w i-A 1$ 等位变异类型 进行了分子检测, 均发现具有 $T a C w i-A 1 a$ 基因型材 料的千粒重显著高于 $T a C w i-A 1 b$ 类型, 进一步验证 了 $T a C w i-A 1 a$ 是粒重的优异等位变异。 $\mathrm{Lu}$ 等 ${ }^{[25]}$ 在普 通小麦 $3 \mathrm{DS}$ 染色体上电子克隆获得了细胞分裂素氧 化酶 TaCKX6a02-D1 基因, 并根据其序列开发共显 性功能标记 TKX3D, 在不同小麦品种能够分子检测 出 TaCKX6a02-D1a 和 TaCKX6a02-D1b 变异类型, 发 现利用该标记能区分小麦粒重的高低, 可满足小麦 粒重高低的分子标记辅助选择需求。之后, 简大为 等 ${ }^{[26]}$ 利用上述功能标记揭示了新疆小麦改良品种与 地方品种的遗传变异, 发现高千粒重等位变异在新 疆改良品种中分布频率明显高于地方品种, 而且大 部分优异等位变异分布频率随着育种时期的推进呈 现不连续性上升的趋势。本研究从基因型的角度来 分析黄淮地区小麦品种(系)粒重的变化情况, 结果 在所有参试材料中共发现 7 种不同的等位变异组合, 且具有不同等位变异组合的品种千粒重存在显著差 异。此外, 没有发现具有 3 个低千粒重等位变异组 合 TaCwi-A1b/TaGS-D1b/TaGw8-B1b 的品种, 这可能 在长期的人工选择过程中低千粒重的劣势等位变异 类型逐渐被淘汰, 也可能是由于本研究所选择试验 材料的代表性存在一定的局限性。

本研究利用小麦粒重功能标记进行分子检测, 并结合其粒重表型测定进行分析, 结果发现少数品 种基因型与千粒重表型不一致的情况, 如具有 2 个 高千粒重和 1 个低千粒重等位变异组合 $T a C w i-A l a$ / $T a G S-D 1 a / T a G w 8-B 1 b$ 品种的千粒重也较低, 这可能 是由于粒重除受上述检测位点控制外, 还存在其他 控制小麦粒重基因位点及其相关基因, 也可能是由 于本试验材料该类型品种数量较少(仅 2 份), 不具有 代表性。从本研究的粒重表型结果来看, 黄淮麦区 品种(系)的千粒重变异范围较大, 且变异系数高, 遗 传变异非常丰富, 具有较大的遗传改良潜力, 也为
篮选较高粒重的优异种质资源创造了有利条件。

\section{4 结论}

利用功能标记分子鉴定的方法对来自于黄淮麦 区的 183 份小麦品种(系)检测, 发现在 $T a C w i-A 1$ 、 $T a G w 8-B 1$ 和 $T a G S-D 1$ 位点上不同等位变异均会导 致小麦千粒重的显著变化, 而 $T a G w 8-B 1$ 和 $T a G S-D 1$ 位点上的等位变异则对小麦粒重的影响更为重要。 参试材料中发现共 7 种不同的等位变异组合, 不同 等位变异组合的品种千粒重存在显著性差异, 没有 发现具有 3 个低千粒重等位变异组合 $T a C w i-A l b /$ $T a G S-D 1 b / T a G w 8-B 1 b$ 的品种, 同时发现具有 3 个高 千粒重等位变异组合 $T a C w i-A 1 a / T a G S-D 1 a / T a G w 8-$ $B 1 a$ 品种的平均千粒重最高, 是优势基因型组合, 所占比例为 $52.5 \%$, 具有这些优势基因型组合的优 异材料将为我国小麦高产育种过程中亲本的选配提 供有效资源。

\section{References}

[1] 刘志勇, 王道文, 张爱民, 梁翰文, 吕慧颖, 邓向东, 葛毅强, 魏珣, 杨维才. 小麦育种行业创新现状与发展趋势. 植物遗传 资源学报, 2018, 19: 430-434.

Liu Z Y, Wang D W, Zhang A M, Liang H W, Lyu H Y, Deng X D, Ge Y Q, Wei X, Yang W C. Current status and perspective of wheat genomics, genetics, and breeding. J Plant Genet Resour, 2018, 19: 430-434 (in Chinese with English abstract).

[2] Zanke C D, Ling J, Plieske J, Kollers S, Ebmeyer E, Korzun V, Argillier O, Stiewe G, Hinze M, Neumann F, Eichhorn A, Polley A, Jaenecke C, Ganal M W, Röder M S. Analysis of main effect QTL for thousand grain weight in European winter wheat (Triticum aestivum L.) by genome-wide association mapping. Front Plant Sci, 2015, 6: 644 .

[3] 宋健民, 戴双, 李豪圣, 程敦公, 刘爱峰, 曹新有, 刘建军, 赵 振东. 山东省近年来审定小麦品种农艺和品质性状演变分析. 中国农业科学, 2013, 46: 1114-1126.

Song J M, Dai S, Li H S, Cheng D G, Liu A F, Cao X Y, Liu J J, Zhao Z D. Evolution of agronomic and quality traits of wheat cultivars released in Shandong province recently. Sci Agric Sin, 2013, 46: 1114-1126 (in Chinese with English abstract).

[4] 曹廷杰, 赵虹, 王西成, 崔党群, 詹克慧. 河南省半冬性小麦品 种主要农艺性状的演变规律. 麦类作物学报, 2010, 30: 439-442.

Cao T J, Zhao H, Wang X C, Cui D Q, Zhan K H. Evolution of main agronomic traits for semi winter wheat varieties in Henan province. J Triticeae Crops, 2010, 30: 439-442 (in Chinese with English abstract).

[5] Ma D Y, Yan J, He Z H, Wu L, Xia X C. Characterization of a cell wall invertase gene $\mathrm{TaCwi}-\mathrm{Al}$ on common wheat chromosome $2 \mathrm{~A}$ and development of functional markers. Mol Breed, 2012, 29: $43-52$.

[6] Jiang Y M, Jiang Q Y, Hao C Y, Hou J, Wang L F, Zhang H N, 
Zhang S N, Chen X H, Zhang X Y. A yield-associated gene TaCWI, in wheat: its function, selection and evolution in global breeding revealed by haplotype analysis. Theor Appl Genet, 2015, 128: 131-143.

[7] Zhang Y J, Liu J D, Xia X C, He Z H. TaGS-D1, an ortholog of rice $O s G S 3$, is associated with grain weight and grain length in common wheat. Mol Breed, 2014, 34: 1097-1107.

[8] Wang S S, Zhang X F, Chen F, Cui D Q. A single-nucleotide polymorphism of TaGS5 gene revealed its association with kernel weight in Chinese bread wheat. Front Plant Sci, 2015, 6: 1166.

[9] Wang S S, Yan X F, Wang Y Y, Liu H M, Cui D Q, Chen F. Haplotypes of the TaGS5-Al gene are its association with high thousand-kernel weight in Chinese bread wheat. Front Plant Sci, 2016, 7: 783.

[10] Hanif M, Gao F M, Liu J D, Wen W E, Zhang Y J, Rasheed A, Xia $\mathrm{X} \mathrm{C}, \mathrm{He} \mathrm{Z} \mathrm{H,} \mathrm{Cao} \mathrm{S} \mathrm{H.} \mathrm{TaTGW6-A1,} \mathrm{an} \mathrm{ortholog} \mathrm{of} \mathrm{rice} \mathrm{TGW6,} \mathrm{is}$ associated with grain weight and yield in bread wheat. Mol Breed, 2016, 36: 1-8.

[11] Hu M J, Zhang H P, Cao J J, Zhu X P, Wang S X, Jiang H, Wu Z Y, $\mathrm{Lu} \mathrm{J}$, Chang C, Sun G L, Ma C X. Characterization of an IAA-glucose hydrolase gene TaTGW6 associated with grain weight in common wheat (Triticum aestivum L.). Mol Breed, 2016, 36: $1-11$.

[12] Yang J, Zhou Y J, Wu Q H, Chen Y X, Zhang P P, Zhang Y, Hu W G, Wang X C, Zhao H, Dong L L, Han J, Liu Z Y, Cao T J. Molecular characterization of a novel TaGL3-5A allele and its association with grain length in wheat (Triticum aestivum L.). Theor Appl Genet, 2019, 132: 1799-1814.

[13] Ma M, Zhao H X, Li Z J, Hu S W, Song W N, Liu X L. TaCYP78A5 regulates seed size in wheat (Triticum aestivum). $J$ Exp Bot, 2015, 67: 1397-1410.

[14] 司文洁，吴林楠，郭利建，周梦蝶，刘香利，马猛，赵惠贤.小 麦粒重相关基因 TaCYP78A5 功能标记开发及验证. 作物学报, 2019, 45: 1905-1911.

Si W J, Wu L N, Guo L J, Zhou M D, Liu X L, Ma M, Zhao H X. Development and validation of the functional marker of grain weight-related gene TaCYP78A5 in wheat (Triticum aestivum L.). Acta Agron Sin, 2019, 45: 1905-1911 (in Chinese with English abstract).

[15] Yan X F, Zhao L, Ren Y, Dong Z D, Cui D Q, Chen F. Genome-wide association study revealed that the TaGW8 gene was associated with kernel size in Chinese bread wheat. Sci Rep, 2019, 9: 2702

[16] 寇程, 高欣, 李立群, 李扬, 王中华, 李学军. 小麦粒重基因 $T a G W 2-6 A$ 等位变异的组成分析及育种选择. 作物学报, 2015, 41: 1640-1647.

Kou C, Gao X, Li L Q, Li Y, Wang Z H, Li X J. Composition and selection of TaGW2-6A alleles for wheat kernel weight. Acta Agron Sin, 2015, 41: 1640-1647 (in Chinese with English abstract).

[17] 时佳，白璐，任毅，穆培源，梁晓东，玛依拉，耿洪伟. 新疆小 麦 $T a G W 2-6 A 、 T a C w i-A 1 、 T a S u s 2-2 B$ 等位变异对粒重的影响及
应用. 分子植物育种, 2018, 16: 848-858

Shi J, Bai L, Ren Y, Mu P Y, Liang X D, Ma Y L, Geng H W. Effects and application of allelic variation of TaGW2-6A, TaCwi-A1 and TaSus $2-2 B$ on grain weight of Xinjiang wheat. Mol Plant Breed, 2018, 16: 848-858 (in Chinese with English abstract).

[18] Chen F, Zhang F Y, Xia X C, Dong Z D, Cui D Q. Distribution of puroindoline alleles in bread wheat cultivars of the Yellow and Huai valley of China and discovery of a novel puroindoline a allele without PINA protein. Mol Breed, 2012, 29: 371-378.

[19] Cui F, Ding A M, Li J, Zhao C H, Li X F, Feng D S, Wang X Q, Wang L, Gao J R, Wang H G. Wheat kernel dimensions: how do they contribute to kernel weight at an individual QTL level. $J$ Genet, 2011, 90: 409-425.

[20] Wu W, Li C J, Ma B L, Shah F, Liu Y, Liao Y C. Genetic progress in wheat yield and associated traits in China since 1945 and future prospects. Euphytica, 2014, 196: 155-168.

[21] Rasheed A, Xia X C, Ogbonnaya F, Mahmood T, Zhang Z W, Mujeeb-Kazi A, He Z H. Genome-wide association for grain morphology in synthetic hexaploid wheats using digital imaging analysis. BMC Plant Biol, 2014, 14: 128.

[22] Gupta P K, Rustgi S, Kumar N. Genetic and molecular basis of grain size and grain number and its relevance to grain productivity in higher plants. Genome, 2006, 49: 565-571

[23] 相吉山, 穆培源, 桑伟, 聂迎彬, 徐红军, 庄丽, 崔风娟, 韩新 年, 邹波. 小麦粒重基因 TaCwi-A1 功能标记 CWI22、CWI21 的验证及应用. 中国农业科学, 2014, 47: 2671-2679.

Xiang J S, Mu P Y, Sang W, Nie Y B, Xu H J, Zhuang L, Cui F J, Han X N, Zou B. Validation and application of function markers CWI22 and CWI21 of TaCwi-A1 gene related to wheat kernel weight. Sci Agric Sin, 2014, 47: 2671-2679 (in Chinese with English abstract).

[24] 刘永伟, 周硕, 王雪征, 孙果忠, 朱金永, 韩秋芬, 李春杰, 赵 和, 王海波. 粒重基因 $T a C w i-A 1$ 等位变异在黄淮麦区小麦品种 (系)中的分布及功能分析. 华北农学报, 2017, 32: 131-137.

Liu Y W, Zhou S, Wang X Z, Sun G Z, Zhu J Y, Han Q F, Li C J, Zhao H, Wang H B. Functional analysis and distribution of allelic variations of $T a C w i-A 1$ gene related to kernel weight in Yellow and Huai River Valleys facultative wheat zone. Acta Agric Boreali-Sin, 2017, 32: 131-137 (in Chinese with English abstract).

[25] Lu J, Chang C, Zhang H P, Wang S X, Sun G L, Xiao S H, Ma C X. Identification of a novel allele of TaCKX6a02 associated with grain size, filling rate and weight of common wheat. PLoS One, 2015, 10: e0144765.

[26] 简大为, 周阳, 刘宏伟, 杨丽, 买春艳, 于立强, 韩新年, 张宏 军, 李洪杰. 利用功能标记揭示新疆小麦改良品种与地方品种 的遗传变异. 作物学报, 2018, 44: 657-671.

Jian D W, Zhou Y, Liu H W, Yang L, Mai C Y, Yu L Q, Han X N, Zhang H J, Li H J. Functional markers reveal genetic variations in wheat improved cultivars and landraces from Xinjiang. Acta Agron Sin, 2018, 44: 657-671 (in Chinese with English abstract). 